\title{
A computer program for testing and analyzing random generation behavior in normal and clinical samples: The Mittenecker Pointing Test
}

\author{
Günter Schulter, Erich Mittenecker, and Ilona Papousek \\ Karl-Franzens-University of Graz, Graz, Austria
}

\begin{abstract}
Random sequence generation tests have proved to be a useful diagnostic tool for the identification of clinically relevant impairments of executive functions and for the study of cognitive functioning in healthy individuals. The most prevalent variety, random number generation, involves several limitations, however. In the original Mittenecker Pointing Test (MPT; Mittenecker, 1958), subjects were instructed to point successively and as randomly as possible at nine unlabeled circles irregularly arranged on a cardboard. With the computer program presented here, Mittenecker's classical test has been transferred to a contemporary format. The MPT can be applied using a standard PC keyboard and computes a series of sophisticated measures of deviations from randomness on the basis of information theory analysis. Because of its easy and well-controlled administration and reduced demands on memory and attention, the automatized MPT offers a wide range of application possibilities in normal but also in severely impaired clinical samples.
\end{abstract}

The generation of random sequences opens up a window to psychological processes that are attributed to the capacity of core components of executive functions (Baddeley, Emslie, Kolodny, \& Duncan, 1998; Knoch, Brugger, \& Regard, 2005; Towse \& Cheshire, 2007). Unlike in most other neuropsychological tests, in random generation tasks, increasing automatization does not reduce the demands on the central executive. In contrast, a continuously high control effort is required to inhibit developing routines (Brown, Soliveri, \& Jahanshahi, 1998; Schmuck \& Wöbken-Blachnik, 1996). In addition, random generation tests are well suited for detecting disturbances in the internal (spontaneous) control of behavior, because no external cues to guide selection of the next response are present (Stoffers, Berendse, Deijen, \& Wolters, 2001). Therefore, random generation tasks are used to identify clinically relevant impairments of executive functions. But in healthy individuals, too, there is substantial interindividual variability in the randomness of produced sequences that seems to be determined by the efficiency of executive (mainly inhibitory) processes. Consequently, random sequence generation is also a useful diagnostic tool rendering sensitive indices of cognitive functioning in the domain of differential psychology (Schulter, 1987).

The most prevalent type of random generation task has been random number generation: For example, subjects are instructed to say the numbers $1-9$ in a random fashion for a number of trials, mostly in synchrony with an external pacing stimulus (e.g., a metronome), in order to control the rate of production. Sometimes letters (or even words) have been used instead of numbers (see Brugger, 1997, and Wa- genaar, 1970, for reviews). However, verbal tasks involve several limitations (see below). Mittenecker (1958) was the first to develop a random motor generation test for the objective assessment of perseverative tendencies that aimed at overcoming these limitations: the Mittenecker Pointing Test (MPT; original German name: Mittenecker Zeigeversuch). In the original MPT, subjects were instructed to point successively, with their index finger and in a sequence as random as possible, at nine unlabeled circles irregularly arranged on a cardboard while the experimenter noted in writing which circles had been tapped. Mittenecker also developed a series of sophisticated measures of randomization performance - that is, quantitative measures of deviations from randomness, which are based on information theory analysis (Mittenecker, 1958, 1960).

The purpose of the present article is to introduce a convenient all-in-one computer program for the automatic and objective assessment of random motor generation behavior. With this software, Mittenecker's (1958) classical behavioral test has been transferred to a contemporary format. The test can be applied using a standard PC and keyboard and automatically calculates several scores of random generation behavior. By publishing the computer program, we would like to draw attention to this alternative random generation task, which has been successfully used since the 1950s, mainly in the German-language literature, and make it available to researchers and clinicians not familiar with the non-English behavioral science tradition.

Most important for random sequence generation is the functionality of inhibitory and memory processes (Miyake et al., 2000). With respect to both executive sub- 
processes, random motor generation tasks like the MPT differ from more classical tasks (random number or letter generation). We propose that, on one hand, performance in the MPT allows more straightforward interpretations of the functionality of these processes than random number generation. On the other hand, analyzing the performance in both modes of generation may be particularly informative in some cases.

In random generation tasks, two natural tendencies must be inhibited: (1) Well-learned response stereotypes cause strong response biases - for instance, the production of numbers and letters according to their natural order (counting up or down, production of letters in alphabetical order), or letters according to patterns occurring in language. Because they are not conducive to the task, they must be permanently suppressed. (2) Since the newly developed selection strategies tend to become routine as well (and, hence, to be perseverated), the building of routines must be suppressed, and developing routines must be permanently inhibited (Schmuck \& Wöbken-Blachnik, 1996). Unlike in classical random number or letter generation tasks, in the MPT, these two processes are not confounded: Tendency 1 is obsolete; only Tendency 2 is assessed. (It is important in this context that in the MPT, the targets are distributed in an irregular pattern; see Figure 1 for an example in which the targets are keyboard keys.) That is, randomization performance in the MPT reflects inhibition of the naturally occurring tendency to repeat already selected sequences (but not sequences that have been overlearned through years of experience). It indicates the extent to which new choices are continuously influenced by previously generated keystrokes and may correspond more directly to the concept of perseveration than does random number generation performance (see also Ginsburg \& Wiegersma, 1991). Scores like the frequently used counting bias in random number generation tasks do not distinguish between inhibition of overlearned sequences and perseverative repetition of sequences, because both may play a role. However, comparison of random motor and random number generation performance in the same subjects may provide information on which of the two inhibitory processes may be primarily impaired.

Both in random number and in motor generation tasks, remembering the frequency with which certain numbers or keys have already been chosen helps to approximate an equal distribution of selected elements (Schmuck \& Wöbken-Blachnik, 1996; Towse \& Cheshire, 2007), but only random number (and letter) generation places an

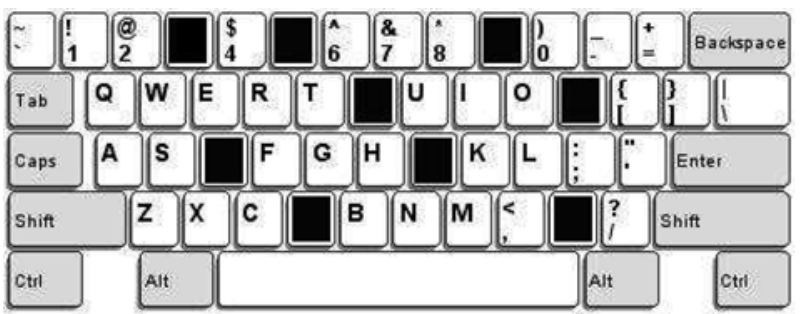

Figure 1. Spatial configuration of keys used in the Mittenecker Pointing Test. additional demand on memory: The item pool must be held ready by short-term memory processes. Especially in patients, the requirement to represent candidate choices is an important source of difficulty in producing random numbers or letters (Towse, 1998). In contrast, in the MPT, the valid response alternatives are permanently visible, and responses outside the valid set of alternatives are not possible. Thus, the additional requirement to exclude responses that obtrude on the subject but are not included in the valid response set does not emerge. This considerably reduces memory load and attentional demands. Consequently, the MPT is also applicable in severely impaired clinical samples. Moreover, the exclusion of trials in which subjects use responses outside the valid response set is obsolete.

Another important difference between the two types of random sequence generation is that in random number and letter generation, sequences are influenced by preexisting individual preferences for and aversions to certain numbers or letters and by the frequency with which letters occur in language (e.g., "e" is more common than "z"; Baddeley, 1966; Kuhl \& Schönpflug, 1974; Mittenecker, 1958). In random motor generation, no such bias exists. Therefore, in contrast to numbers or letters, the relative frequencies of selected (unlabeled) keys are virtually equal in most healthy individuals (Mittenecker, 1958). This improves the definiteness of some measures of randomization performance. The dependence of individual differences in the inequality of relative frequencies of numbers or letters from the strength of individual preferences has led some researchers to interpret them as an expression of superstitious behavior rather than as an indicator of an executive deficit (e.g., Avons, Nunn, Chan, \& Armstrong, 2003).

In contrast to classical random number and letter generation tasks, speaking is not required in random motor generation. Consequently, the MPT is also applicable in aphasic patients or in patients with speech problems. In addition, the independence of speech makes random motor generation tasks especially well suited for using them concurrently with other tasks involving verbal output. The ability to perform two tasks simultaneously is a key method of studying the central executive (Baddeley et al., 1998), and, thus, their use in dual-task studies seems to be one of the most gainful applications of random generation tasks. In addition, the test includes the option to use either one or both hands, which makes the test also useful in studies on functional hemispheric asymmetries (e.g., Schulter, 1987).

A more pragmatic problem of random generation tasks is the need to record and encode the subjects' responses, a relatively laborious and potentially error-prone task. The MPT computer program presented here features automatic and objective collection of data, as well as calculation of several sophisticated scores, in an all-in-one computer program. Another advantage is the standardized administration of the task.

\section{THE MPT COMPUTER PROGRAM}

The MPT software program, developed employing Delphi (Object Pascal), runs under Windows 2000, 
XP, and Vista. After the program file MPT.exe has been downloaded, ${ }^{1}$ extracted, and stored on the hard disk of a computer, the software can be installed by doubleclicking the MPT.exe file. The computer keyboard has to be prepared by covering all keys except those needed for the task. (A cover can easily be made, e.g., of the plastic keyboard cover that usually comes with PCs.) The following nine keys of the standard Windows keyboard must be accessible (U.S. layout): "3," "5," "9," "Y," "P," "D," "J," "V," and "." (German layout: "3," "5," "9,", "Z," "P," "D," "J," "V," and ".") (see Figure 1). The labels of these nine keys must be concealed with adhesive stickers. Once the software is opened by double-clicking the MPT icon, the screen shows three buttons: The "Start" button is for defining the settings of a test session and subsequent administration of the test. The "Information" button gives a summary of the test scores that will be stored in the output file. Clicking the "Quit" button will close the program.

\section{Settings}

After the user clicks the "Start" button, some input boxes will appear: A "Subject ID" has to be typed in so that the user will later be able to identify the test results of an individual (e.g., a serial number). If multiple test sessions are planned, " 1 " must be typed into the "Session" box for the first test session of an individual, "2" for the second test session, and so on. These data are used to define the name of the output file: The test scores of Subject "100" (Subject ID) in Session "1" (Session) will be stored in the file "MPT100_1.dat," located in a subfolder called "IMPT_data." The output files can be opened and read with any text editor, and the data can easily be imported into statistical software packages for further analysis.

The following test settings can be modified: number of sequences to be produced within one session ("Number of sequences," 1-10); total number of keystrokes to complete one sequence ("Length of sequence," 1-1,000); and time lag between sequences, if the production of more than one sequence will be required ("Interval between sequences," $1-900 \mathrm{sec}$ ). If the box "Pacing stimulus" is checked, an auditory pacing stimulus will be given; the number of stimuli per second can be entered in the input box labeled "Key strokes per second" with allowed values in the range of 0.1-3.0; if the box is unchecked, the test subject is free to choose his or her own response speed. Finally, it can be chosen whether detailed information on produced dyads and selection frequencies for single keys will be stored in the output file in addition to the calculated test scores ("Save dyad matrix and relative key selection frequency").

\section{Execution of the Test}

After the subject clicks the "OK" button in the settings window, text will be presented on the screen instructing the subject to press the available keys in a completely random fashion. If pacing stimuli were selected in the settings, instructions to follow the pace will also be given. Clicking the "OK" button again will close the instruction window. The following screen shows the numbers of the current session and sequence. The test run can be stopped at this stage by clicking the "Cancel" button. After the "Start" button has been clicked, the test will begin with the subject's pressing the first key and will end with the last of the predefined number of keystrokes ("Length of sequence"). Then, if more than one sequence per session has been selected in the settings window ("Number of sequences"), the screen will show the word "pause" until the predefined time between sequences ("Interval between sequences") has passed, and the next sequence will be started automatically. After the production of the last scheduled sequence, the initial window reappears, and the program is ready for testing the same subject in an additional session (e.g., under another condition) or for testing the next study subject.

\section{Measures of the MPT}

Deviations from true random series are due to perseveration tendencies leading the individual to repeat sequences composed of several items. This observation gave direction to the development of Mittenecker's test and its measures of randomization behavior (Mittenecker, 1958, 1960). In the following section, the definitions of these measures are given, along with their cognitive meaning and evidence of their validity.

Symbol redundancy. Symbol redundancy refers to the inequality of the relative frequencies of chosen keys. According to information theory analysis (Shannon \& Weaver, 1949), the randomness of a series decreases and redundancy increases with increasing deviation of the frequency distribution of chosen response alternatives from equality.

The relative response frequency $p(i)$ is defined as the frequency $(f)$ of pressing the key $i$, related to the total number of keystrokes $(N)$ in a given sequence:

$$
p(i)=\frac{f(i)}{N} .
$$

Using a response set of $n$ different alternatives (in the case of the MPT, $n=9$ ), the symbol entropy or average symbol information $(\bar{H})$ is defined as

$$
\bar{H}=-\sum_{i=1}^{n} p(i) \log _{2} p(i) .
$$

In a given sequence, symbol information is maximal when all alternatives were chosen with equal frequency. $H_{\max }$ is calculated as

$$
H_{\text {max }}=\log _{2} n .
$$

The amount of symbol information provided by a given sequence, then, is the average symbol information related to the maximum information possible (relative entropy). Accordingly, the symbol redundancy, $S R$, is defined as the complement of relative entropy:

$$
S R=1-\frac{\bar{H}}{H_{\max }} .
$$

That is, if information is maximal, redundancy is minimal, and the series approximates randomness or maximal disorder. With increasing redundancy (when frequencies 
$f(i)$ are increasingly unequal), the predictability of the series of responses increases.

Symbol redundancy is equivalent to the so-called $R$ score. Latent variable analysis suggests that it is related to the memory-updating component of random sequence generation (Miyake et al., 2000). The measure's relationship to memory is supported by empirical findings showing that applying the MPT with a memory aid (display of the chosen keys on the computer monitor) reduces the symbol redundancy of a produced series (Herzog, 1989). In random number generation, the contribution of memory to $S R$ is less unequivocal, because strong individual preferences may play a major role, which may exceed that of differences in memory processes (e.g., Avons et al., 2003).

Context redundancy. Whereas $S R$ only refers to the balanced selection of single keys, in context redundancy $(C R)$, all possible pairs of keys (dyads) are taken into account. $C R$ reflects the extent to which responses are continuously influenced by previously chosen alternatives. In long, true random series, all possible dyads (and triads, etc.) are approximately equiprobable, but in series produced in random sequence generation tasks, the frequency distribution of dyads typically deviates from equality to a considerable extent.

$\mathrm{CR}_{1}$ is defined as the complement of the average context information - that is, the average information of all symbols (keys) $j$, given the knowledge that the immediately preceding key was $i$. According to information theory, context information is composed of the average information of dyads $(i, j)$ - that is, $\left[-\sum_{i=1}^{n} \sum_{j=1}^{n} p(i, j) \log _{2} p(i, j)\right]$ and the average information of single keys $(i)$ - that is, $\left[-\sum_{i=1}^{n} p(i) \log _{2} p(i)\right]$ - and is related to the maximum information possible, $\mathrm{H}_{\max }$ (Mittenecker \& Raab, 1973). Consequently, context redundancy (the redundancy of dyads) is defined as

$$
C R_{k}=1-\frac{\left[-\sum_{i=1}^{n} \sum_{j=1}^{n} p(i, j) \log _{2} p(i, j)\right]-\left[-\sum_{j=1}^{n} p(i) \log _{2} p(i)\right]}{H_{\max }},
$$

where, for the moment, $k=1$, to indicate dyads of immediately adjacent responses. Below, we discuss other forms of dyads, where $k$ can take different integer values. In $C R_{1}$, only pairs of adjacent responses are taken into account. It is based on the relative frequencies with which two specific keys were selected in adjacent responses. Computational routines of the MPT also calculate redundancies of longer sequences $\left(C R_{2}-C R_{6}\right)$. However, because it would be inappropriate to calculate context information measures for $9 \times 9 \times 9=729$ different triads (or even more possible combinations for longer sequences) from a sample of a few hundred responses, a different approach is followed in the calculation of the redundancies of longer sequences $(k=$ $2-6)$ : They are approximated by calculating the context redundancies of dyads, defining dyads $(i, j)$ as pairs of elements that were not chosen in adjacent responses, but in responses separated by $1-5$ other responses. Therefore, for the calculation of $C R_{1}$, the frequency of dyad $(i, j)$ is defined as the frequency with which the keys $i$ and $j$ were chosen in adjacent responses $(k=1)$. For the calculation of $C R_{2}$, instead, the frequency of dyad $(i, j)$ is defined as the frequency with which the keys $i$ and $j$ were selected in responses with one response in between them - that is, in one response and a response following the next response $(k=2)$. For $k=3$, dyad $(i, j)$ is defined as the frequency of the pair of keys $i$ and $j$ in responses separated by two other responses, and so on. The equation for $C R_{k}$ is the same for these cases as that for $C R_{1}$. Only the definition of the dyad changes across the conditions.

In most cases, $C R_{1}$ seems to suffice for group comparisons (e.g., Schulter, 1987), but there is some evidence that indices of dependencies based on two responses with several other responses in between $\left(C R_{2}-C R_{6}\right)$ may supply interesting results in the domain of differential psychology (e.g., Guttmann, 1966; Remschmidt, 1970).

Context redundancy was used by Mittenecker (1958, 1960) in the evaluation of his earliest MPT data and later on by other authors-for instance, Tulving (1962). For the calculation of his measure of subjective organization, Tulving used a formula equivalent to that for $C R_{1}$ but based on absolute (instead of relative) frequencies. Tulving's formula was then adopted by Evans (1978), who called it the random number generation score. A simpler version of the measure is the so-called Null-Score, which is the number of dyads of consecutive responses never named by the subject (Guttmann, 1967).

Empirical studies of the MPT reveal that the major part of the interindividual variance in context redundancy can be attributed to the tendency to repeat certain sequences of responses en bloc (Mittenecker, 1960). There is substantial evidence for context redundancy as an indicator of the efficiency of inhibitory processes. For instance, context redundancy increases with faster response rates (e.g., Herzog, 1989). This was interpreted as a loss of control over perseveration, which corresponds to the more recent concepts of flexibility and inhibitory control (Miyake et al., 2000). A number of researchers have used the original (not yet computerized) MPT to study psychiatric and neurological disorders, such as schizophrenia (Axmacher, Bente, \& Ferner, 1970; Mittenecker, 1953, 1960; Morrens, Hulstijn, Lewi, De Hert, \& Sabbe, 2006; Morrens, Hulstijn, Lewi, \& Sabbe, 2008; Morrens, Hulstijn, \& Sabbe, 2006), Parkinson's disease (Stoffers et al., 2001; Stoffers et al., 2007), and epilepsy (Remschmidt, 1970, 1973; Ruser, 1990), as well as individual differences and intraindividual variations in healthy samples (Krug, Mölle, Dodt, Fehm, \& Born, 2003; Krug, Stamm, Pietrowsky, Fehm, \& Born, 1994; Perras, Smolnik, Fehm, \& Born, 2001; Schulter, 1987). Together, the findings confirmed that higher context redundancy was related to restricted cognitive flexibility. MPT performance in the clinical groups corresponded to the inability to change cortical behavioral programs in situations requiring the internal regulation of behavior that has been described in these conditions (Stoffers et al., 2001). The findings of Schulter (1987) were in accordance with evidence indicating primary involvement of the left lateral frontal cortex in inhibition-related cognitive functions such as overriding a highly activated representation (e.g., Hirshorn \& Thompson-Schill, 2006; Jonides \& Nee, 2006). 
Context redundancy proved to be a particularly sensitive measure in these studies. For instance, the negative impact of faster production rates and of additional stimulation on randomization performance was considerably greater for context redundancy than for symbol redundancy (Schulter, 1987; Schwibbe, 1985). Mittenecker (1960) showed that groups of neurotic and schizophrenic patients and healthy individuals differed considerably in context redundancy but not in symbol redundancy and other MPT measures. In normal conditions, symbol redundancy and context redundancy are not correlated (Herzog, 1989; Mittenecker, 1960; Schwibbe, 1985).

Coefficient of constraint. This measure $\left(C C_{1}-C C_{6}\right)$ is similar to context redundancy, but it additionally takes the deviations from equality of the frequency distribution of single keys into account.

As opposed to $C R_{k}$, the dyad entropy underlying the calculation of $C C_{k}$ is not related to the maximum symbol entropy but to the actual symbol entropy:

$$
C C_{k}=2-\frac{\sum_{i=1}^{n} \sum_{j=1}^{n} p(i, j) \log _{2} p(i, j)}{\sum_{j=1}^{n} p(i) \log _{2} p(i)} .
$$

$C C_{1}-C C_{6}$ (for step sizes from $k=1$, i.e., dyads based on adjacent responses, to $k=6$, i.e., dyads based on two responses with five other responses in between) are calculated.

In the MPT, the correlation between coefficients of constraint and context redundancy is usually close to $r=$ 1.0 (Herzog, 1989), because the two measures only differ if the distribution of selected keys is not equal. As opposed to random number or letter generation tasks, in the MPT, the distribution of single keys is close to equal in most groups of subjects (Mittenecker, 1960).

Frequency distribution of repetition distances (gaps). The gap lengths between the stroke of a key and the next stroke of the same key are measured in terms of the number of strokes of other keys in between. One common bias in random sequence generation is that subjects tend to avoid immediate repetitions of the same response alternatives. This repetition avoidance bias is a well-known phenomenon for immediately consecutive responses, but it has also been observed for more distant responses; that is, individuals were also shown to avoid repetition of response alternatives produced up to six responses earlier (Chapanis, 1995; Kuhl \& Schönpflug, 1974; Teraoka, 1963; Wagenaar, 1970). In the MPT, frequencies of repetitions for step sizes from $k=1$ ( $G_{1}$; i.e., the number of immediate repetitions corresponding to conventional repetition avoidance measures) to $k=20\left(G_{20}\right)$ are available. This may be illustrated by the following keystroke series: $13514534434 \ldots$ The first repetition to be recorded is that of " 1 "; its step size is 3 (third stroke after initial stroke of "1"). The next repetition to be recorded will be that of "3," with four other keystrokes in between (i.e., $k=5$ ), and so on. All of the distances are entered in a frequency of distances distribution vector (starting with distance $k=1$ ) and are stored in the output file.
Median of repetition gap distribution (MdG). The median of the frequency distribution of repetition distances has been used as an indicator of the so-called cycling bias. Cycling refers to the fact that subjects tend to make every possible response before one response alternative is repeated (Ginsburg \& Karpiuk, 1994). $M d G$ is low in individuals with a strong tendency to repeat responses in short intervals. Miyake et al. (2000) attributed this score to the memory-updating component of random sequence generation.

In the MPT program, the first and the third quartile of the repetition gap distribution are also provided (repetition gap scores of 1 and 3), which can be used to calculate a nonparametric measure of variability (interquartile range).

Response rate. If the generation of a sequence was not paced, the response rate can be calculated as the arithmetic mean of the time intervals $t_{a}$ (in seconds) between two consecutive keystroke responses $a-1$ and $a$. Given $N$ keystrokes, $N-1$ time intervals are taken into account.

$$
R R=\frac{1}{N-1} \sum_{a=2}^{N} t_{a} .
$$

The variance of time intervals between the keystroke responses is calculated as

$$
s^{2}=\frac{1}{N-2} \sum_{a=2}^{N}\left(t_{a}-R R\right)^{2} .
$$

In the MPT, there are remarkable interindividual differences in the self-selected speed of responding and substantial correlations between response rate and the degree of randomness of produced sequences (Herzog, 1989; Schulter, 1987). The analysis of self-selected response rates is an alternative way to describe the relationship between production rate and random generation performance, which has been reported using random number or letter generation tasks (Baddeley et al., 1998; Hamdan, de Souza, \& Bueno, 2004; Jahanshahi, Saleem, Ho, Dirnberger, \& Fuller, 2006).

If a study is not aimed at investigating the effects of different response rates, the production rate must be controlled by a pacing signal in order for individual differences in the randomization measures to be interpretable.

Lateral preference. In addition to Mittenecker's original scores of random generation behavior, the nowcomputerized MPT calculates a laterality coefficient relating the frequency of selected keys in the left to that in the right hemispace. That is, the selection frequencies of the four keys on the left side of the keyboard (i.e., keys "3," " 5 ," "D," and "V" $\left.\left[f_{L}\right]\right)$ are related to the four keys on the right (i.e., keys " 9 ," "P," "J," and “.” $\left[f_{R}\right]$ ), excluding the "Y" key on an English keyboard and the "Z" key on a German keyboard positioned in the center of all of the keys used.

$$
L Q=\frac{f_{L}-f_{R}}{f_{L}+f_{R}} .
$$

The score may add information about hemispatial inattention without the necessity of applying another test. Given the promising findings with the MPT in psychiatric 
Table 1

Structure of the Output File

\begin{tabular}{lc}
\hline \multicolumn{1}{c}{ Parameter } & Number of Values \\
\hline Length of sequence & 1 \\
Symbol redundancy & 1 \\
Context redundancy & 6 \\
Coefficient of constraint & 6 \\
Repetition distances & 20 \\
Repetition gap scores & 3 \\
Response rate & 1 \\
Variance of time intervals & 1 \\
Lateral preference & 1 \\
Optional: & \\
Matrix of dyads & $9 \times 9$ \\
Key selection frequencies & 9 \\
\hline
\end{tabular}

and neurologic conditions associated with a systematic underrepresentation of the right side of space (particularly schizophrenia; Harvey, Nelson, Haller, \& Early, 1993; Michel et al., 2007), this may be a useful additional feature of the random motor generation test.

If the lateral preference score is used, it is essential to make sure that the keyboard is centered in front of the subject during the task; that is, the imagined line between the "G" and " $\mathrm{H}$ " keys should point to the navel of the test subject.

Matrix of dyads and key selection frequencies. Additional information may be drawn from the total matrix of (first-order) dyad frequencies. Therefore, the matrix of dyads per sequence is also stored in the output file - namely, in the following order: Key $1 \times$ Keys 1-9, Key $2 \times$ Keys 1-9, Key $3 \times$ Keys 1-9, and so on. Finally, the selection frequencies for each of the nine keys are listed in the following order of keys: "3," "5," "9," "Y" (“Z”), "P," “D," “J," "V," “.”.
Structure of the output file. The different measures are listed in the output file in the order given in Table 1.

\section{VALIDATION OF THE MPT COMPUTER PROGRAM}

In order to verify the software program, the data from two independent studies using the computerized MPT were compared with the data from a study in which the original (physical) version of the test was used in similar conditions. In addition to the means and standard deviations of the MPT measures, their retest reliabilities and interrelationships were evaluated.

\section{Samples and Methods}

Reference study. Comparison data were obtained from a study by Herzog (1989). In this study, the original MPT (not computerized) was used in a sample of 101 subjects from different occupational groups, with the majority being students ( 50 women, 51 men). Their ages were between 18 and 45 years. The MPT was applied with a pacing stimulus (1.8 responses per second) and a total of 180 responses were required.

Study 1 . One hundred twenty-three university students from various disciplines participated in Study 1 (64 women, 59 men). Their ages were between 18 and 31 years. The MPT computer program was applied in two separate sessions, 2-4 weeks apart, using the following settings: keystrokes per second $=1.2$, number of keystrokes $=180$.

Study 2. One hundred two university students, 18-48 years old, (52 women, $50 \mathrm{men}$ ), who were predominantly psychology undergraduates, took part in the second study in which the MPT computer program was used. In this study, the response rate was 1 keystroke per second, and the number of keystrokes was again 180 .

\section{Results and Discussion}

The means and standard deviations of the MPT measures obtained in the three studies are shown in Table 2.

Table 2

Means and Standard Deviations of MPT Measures Obtained With the Original and the Computerized Forms of the Test

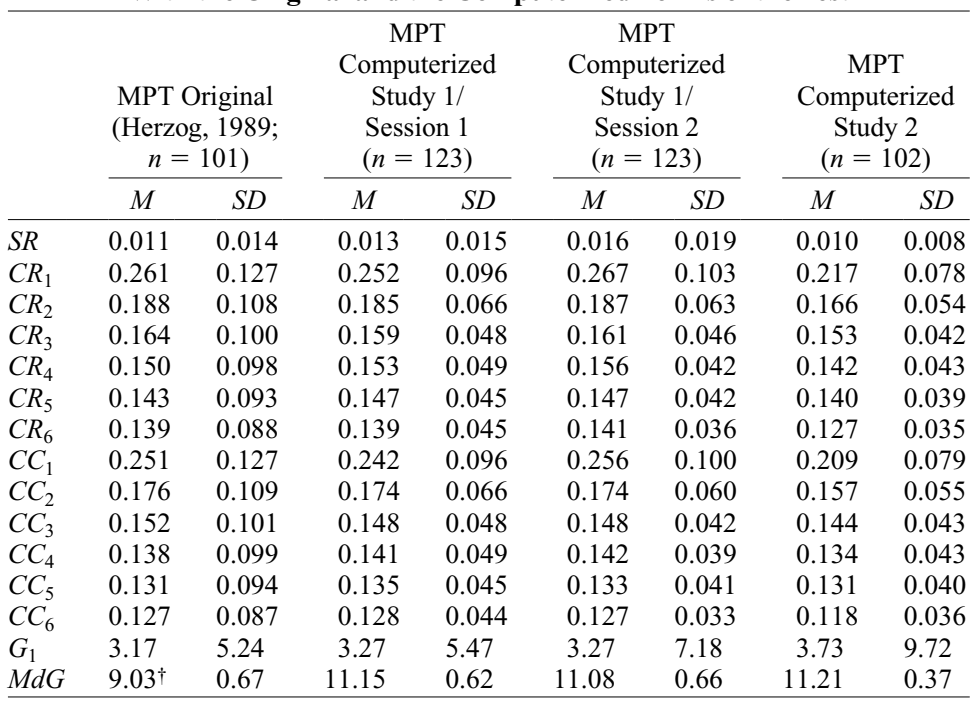

$\dagger$ The mean $M d G$ in Herzog (1989) is not directly comparable with the mean $M d G$ in the computer test (see the text). 
They show that the classical and the computerized form of the test produced very similar data. The means of the information theoretical measures $S R, C R$, and $C C$ obtained with the computer test were evidently in the same range as those obtained with the original method. The same holds true for the number of immediate repetitions $\left(G_{1}\right)$. Minor deviations can be attributed to small variations in the production rate and composition of the samples between the studies (slightly better scores in Study 2, with a lower production rate and a homogeneous sample of psychology students). Particularly, the means of the inhibition-related measure $C R$, which has been proved to be the most sensitive measure in past studies (Mittenecker, 1960; Schulter, 1987; Schwibbe, 1985), were virtually identical in Study 1 (computer test, $C R_{1}=.252$, Session 1, and $C R_{1}=.267$, Session 2) and the reference study (original test, $C R_{1}=.261$ ). The mean value of $M d G$ in the reference study is not directly comparable with those of the computer test; because of a special research question, a slightly different computational approach was used in Herzog (1989). In addition to the means, retest correlations and intercorrelations among the MPT measures provided further evidence for the validity of the MPT computer program.

The retest reliabilities (Study 1) for $S R, C R_{1}$, and $C C_{1}$ were $r=.76(p<.001), r=.75(p<.001)$, and $r=$ $.75(p<.001)$, respectively. The retest reliabilities for the number of immediate repetitions $\left(G_{1}\right)$ and the median of the repetition gap distribution $(M d G)$ were $r=.36(p<$ $.001)$ and $r=.43(p<.001)$, respectively. The retest reliabilities of the information theoretical measures $S R$, $C R_{1}$, and $C C_{1}$ obtained with the computer test were in accordance with scores that were reported for $C R_{1}$ in the classical (noncomputerized) MPT $(r=.66-.86$; interval, 1-15 days; Schwibbe, 1985). Somewhat lower temporal stabilities have been reported for $S R$ in the classical, noncomputerized MPT ( $r=.38-.88$; Schwibbe, 1985). The retest reliabilities of the information-theoretical scores in the computer test were in a range that is common for behavioral tests. They indicate that $S R$ and $C R_{1}$ are about equally determined by inter- and intraindividual differences and, thus, are suitable to assess both temporally stable individual differences (traits) and situational (state) effects. The more simple scores, $G_{1}$ and $M d G$, showed only low temporal stability and do not seem to be suitable to assess temporally stable characteristics of healthy individuals.

The intercorrelations among MPT measures are reported as the average correlations using the data of Studies 1 and 2. According to the view that $S R$ and $M d G$ are predominantly related to the memory-updating component of random sequence generation, whereas $C R$ mainly reflects the efficiency of inhibitory processes, $S R$ and $M d G$ should be correlated with each other but not with $C R$. The results obtained with the computer form of the MPT corresponded to this pattern: $S R$ with $M d G, r=-.25$ ( $p<$ $.01) ; S R$ with $C R_{1}, r=.14$ (n.s.); $M d G$ with $C R_{1}, r=-.06$ (n.s.). This result is in accordance with those of studies in which the original form of the test was used, in which $S R$ was also negatively correlated with $M d G$, whereas $C R_{1}$ was not correlated with $S R$ or with $M d G$ (Herzog, 1989; Mittenecker, 1960; Schwibbe, 1985). As in the reference study, the correlation between $C R_{1}$ and $C C_{1}$ was close to $1.0(r=.99)$.

In order to evaluate whether randomization performance in the MPT may be affected by repeated performance or practice, a multivariate ANOVA was conducted with session (1,2; Study 1) as a within-subjects factor and $S R, C R_{1}, C C_{1}, G_{1}$, and $M d G$ as the dependent variables $[F(5,118)=1.7$, n.s.]. No change of randomization performance after repeated performance of the test had also been shown for the noncomputerized version of the MPT (Schwibbe, 1985). Consequently, the MPT is highly suitable for repeated use. Unlike other neuropsychological tests measuring similar functional processes (e.g., the Wisconsin Card Sorting Test), the validity of the MPT is not affected by repeated application (Schwibbe, 1985).

\section{CONCLUSION}

The MPT computer program produces data that are equivalent to the original (noncomputerized) version of the test. Because of the special features of random motor generation and its easy and well-controlled administration, the automatized MPT offers a wide range of application possibilities in healthy and clinical samples.

Since the development of the original MPT, other authors have suggested alternatives to the classical verbal random generation tasks, also involving motor responses (e.g., Annoni \& Pegna, 1997; Baddeley, 1996; Hoffmann et al., 2003; Schneider, Joppich, van der Lugt, Däuper, $\&$ Münte, 2004). We prefer the MPT because it does not use labeled response sets, which may confound random number generation with random motor generation. The MPT program also avoids spatially ordered layouts of the available keys, which can cause problems similar to those for labeled response sets.

Towse and Neil (1998) introduced a Windows 95 program for calculating a number of measures of random generation behavior, with special focus on random number generation. Towse and Neil's program provides some special measures that are only relevant in random number generation and, hence, are not included in the MPT program (adjacency, turning point index, phase length, runs, first-order difference). Thus, if biases more directly related to counting are the focus of interest, random number generation and Towse and Neil's program may be a good choice. The MPT program differs from that of Towse and Neil by being focused on random motor generation. It provides an all-in-one system for measuring and analyzing random motor generation. In addition to normal populations, such a system may be especially useful for situations where random number generation is not feasible (e.g., the absence of speech) and for severely impaired clinical populations.

\section{AUTHOR NOTE}

The authors are grateful to Bernhard Weber for valuable assistance in developing the computer program. The development of the program was 
partly supported by the Austrian National Bank (Jubiläumsfonds), Project 7406. Correspondence concerning this article should be addressed to G. Schulter, Karl-Franzens-University of Graz, Department of Psychology, Univ.-Platz 2, A-8010 Graz, Austria (e-mail: guenter.schulter@, uni-graz.at).

\section{REFERENCES}

Annoni, J.-M., \& Pegna, A. J. (1997). Random motor generation in a finger tapping task: Influence of spatial contingency and of cortical and subcortical hemispheric brain lesions. Journal of Neurology, Neurosurgery, \& Psychiatry, 63, 654-659.

Avons, S. E., Nunn, J. A., Chan, L., \& Armstrong, H. (2003). Executive function assessed by memory updating and random generation in schizotypal individuals. Psychiatry Research, 120, 145-154.

Axmacher, I., Bente, D., \& Ferner, U. (1970). Informationsstatistische Untersuchungen zur Struktur einfacher Handlungsfolgen bei endogenen Psychosen [Information statistical study on the structure of simple action sequences in major psychoses]. Arzneimittel Forschung, 20, 919-921.

BADDELEY, A. D. (1966). The capacity for generating information by randomization. Quarterly Journal of Experimental Psychology, 18, 119-129.

BAdDELEy, A. D. (1996). Exploring the central executive. Quarterly Journal of Experimental Psychology, 49A, 5-28.

Baddeley, A. D., Emslie, H., Kolodny, J., \& Duncan, J. (1998). Random generation and the executive control of working memory. Quarterly Journal of Experimental Psychology, 51A, 819-852.

Brown, R. G., Soliveri, P., \& Jahanshahi, M. (1998). Executive processes in Parkinson's disease-Random number generation and response suppression. Neuropsychologia, 36, 1355-1362.

BRUGGER, P. (1997). Variables that influence the generation of random sequences: An update. Perceptual \& Motor Skills, 84, 627-661.

Chapanis, A. (1995). Human production of "random" numbers. Perceptual \& Motor Skills, 81, 1347-1363.

Evans, F. J. (1978). Monitoring attention deployment by random number generation: An index to measure subjective randomness. Bulletin of the Psychonomic Society, 12, 35-38.

GinsBURG, N., \& KARPIUK, P. (1994). Random generation: Analysis of the responses. Perceptual \& Motor Skills, 79, 1059-1067.

Ginsburg, N., \& WiEgERSMA, S. (1991). Response bias and the generation of random sequences. Perceptual \& Motor Skills, 72, 1332-1334.

Guttmann, G. (1966). Komplexe Ordnungstendenzen in Verhaltensabläufen und ihre differentialdiagnostische Bedeutung. Zeitschrift für Experimentelle und Angewandte Psychologie, 13, 19-30.

GutTmann, G. (1967). Die empirische Erprobung von Methoden zur Überprüfung der Ergodizität von Ereignisfolgen [Empirical testing of methods for the examination of the ergodicity of sequences]. Archiv für die gesamte Psychologie, 119, 251-260.

Hamdan, A. C., De Souza, J. A., \& Bueno, O. F. (2004). Performance of university students on random number generation at different rates to evaluate executive functions. Arquivos de Neuropsiquiatria, 62, 58-60.

Harvey, S. A., Nelson, E., Haller, J. W., \& Early, T. S. (1993). Lateralized attentional abnormality in schizophrenia is correlated with severity of symptoms. Biological Psychiatry, 33, 93-99.

HerzoG, G. R. (1989). Die Zufallskonzepttheorie: Experimente zur Vorstellung vom Aufbau von Zufallsreihen [The random concept theory: Experiments on the idea of random series]. Zeitschrift für experimentelle und angewandte Psychologie, 36, 199-220.

HiRshorn, E. A., \& Thompson-SchiLl, S. L. (2006). Role of the left inferior frontal gyrus in covert word retrieval: Neural correlates of switching during verbal fluency. Neuropsychologia, 44, 25472557.

Hoffmann, E., Servais, L., Campanella, S., Stillemans, E., LEWI, P., \& JANSSEN, P. (2003). Use of the stereotypy test apparatus with severe juvenile offenders. [Abstract] Schizophrenia Research, 60 (Suppl. 1), 171.

Jahanshahi, M., Saleem, T., Ho, A. K., Dirnberger, G., \& Fuller, R. (2006). Random number generation as an index of controlled processing. Neuropsychology, 20, 391-399.

JoNIDES, J., \& NEE, D. E. (2006). Brain mechanisms of proactive interference in working memory. Neuroscience, 139, 181-193.
Knoch, D., Brugger, P., \& Regard, M. (2005). Suppressing versus releasing a habit: Frequency-dependent effects of prefrontal transcranial magnetic stimulation. Cerebral Cortex, 15, 885-887.

Krug, R., Mölle, M., Dodt, C., Fehm, H. L., \& Born, J. (2003). Acute influences of estrogen and testosterone on divergent and convergent thinking in postmenopausal women. Neuropsychopharmacology, 28, 1538-1545.

Krug, R., Stamm, U., Pietrowsky, R., Fehm, H. L., \& Born, J. (1994). Effects of menstrual cycle on creativity. Psychoneuroendocrinology, 19, 21-31.

KuHL, J., \& SchÖNPFLUG, W. (1974). Ordnungsverhalten, Lärmbelästigung und Persönlichkeit [Stereotypy, noise pollution, and personality]. Psychological Research, 37, 143-176.

Michel, C., Cavezian, C., D’Amato, T., Dalery, J., Rode, G., Saoud, M., \& Rossetti, Y. (2007). Pseudoneglect in schizophrenia: A line bisection study with cueing. Cognitive Neuropsychiatry, 12, 222-234.

Mittenecker, E. (1953). Perseveration und Persönlichkeit. 1. Teil: Experimentelle Untersuchungen [Perseveration and personality, Part 1: Experimental studies]. Zeitschrift für Experimentelle und Angewandte Psychologie, 1, 5-31.

MitTENeCKer, E. (1958). Die Analyse "zufälliger" Reaktionsfolgen [The analysis of "random" action sequences]. Zeitschrift für Experimentelle und Angewandte Psychologie, 5, 45-60.

MitTENECKER, E. (1960). Die informationstheoretische Auswertung des Zeigeversuchs bei Psychotikern und Neurotikern [The information theoretical analysis of the pointing test applied in psychotics and neurotics]. Zeitschrift für Experimentelle und Angewandte Psychologie, 7, 392-400.

MitTEneCKer, E., \& RAAB, E. (1973). Informationstheorie für Psychologen [Information theory for psychologists]. Göttingen: Hogrefe.

Miyake, A., Friedman, N. P., Emerson, M. J., Witzki, A. H., HowERTER, A., \& WAGER, T. (2000). The unity and diversity of executive functions and their contributions to frontal lobe tasks: A latent variable analysis. Cognitive Psychology, 41, 49-100.

Morrens, M., Hulstijn, W., Lewi, P. J., De Hert, M., \& Sabbe, B. G. C. (2006). Stereotypy in schizophrenia. Schizophrenia Research, 84, 397-404.

Morrens, M., Hulstijn, W., Lewi, P. J., \& Sabbe, B. G. C. (2008). Bleuler revisited: Psychomotor slowing in schizophrenia as part of a catatonic symptom cluster. Psychiatry Research, 161, 121-125.

Morrens, M., Hulstijn, W., \& SabBe, B. G. C. (2006). Stereotypy in schizophrenic patients on atypical antipsychotics versus conventional neuroleptics. European Neuropsychopharmacology, 16, S392-S393.

Perras, B., Smolnik, R., Fehm, H. L., \& Born, J. (2001). Signs of sexual behaviour are not increased after subchronic treatment with LHRH in young men. Psychoneuroendocrinology, 26, 1-15.

Remschmidt, H. (1970). Experimentelle Untersuchungen zur sogenannten epileptischen Wesensveränderung [Experimental studies on the so-called epileptic character change]. Fortschritte der Neurologie und Psychiatrie, 38, 524-540.

Remschmidt, H. (1973). Psychological studies of patients with epilepsy and popular prejudice. Epilepsia, 14, 347-356.

RUSER, I. (1990). Klinische und testpsychologische Untersuchungen zur Psychopathologie der Epilepsien [Clinical and psychological test studies of the psychopathology of epilepsy]. Psychiatrie, Neurologie und medizinische Psychologie, 42, 394-405.

SCHMUCK, P., \& WöBKEn-BlachniK, H. (1996). Verhaltensflexibilität und Arbeitsgedächtnis [Behavioral flexibility and working memory]. Diagnostica, 42, 47-66.

SChNeIder, S., Joppich, G., VAn der Lugt, A., DëUPer, J., \& MÜNTE, T. F. (2004). Brain potentials and self-paced random number generation in humans. Neuroscience Letters, 367, 51-55.

Schulter, G. (1987). Funktionale Asymmetrien der Großhirnhemisphären als Determinanten der Perseverationstendenz [Functional asymmetries of the cortical hemispheres as determinants of perseveration]. In E. Raab \& G. Schulter (Eds.), Perspektiven psychologischer Forschung [Perspectives of psychological research] (pp. 77-95). Vienna: Deuticke.

ScHWiBBE, M. H. (1985). Experimentelle Untersuchungen zur Eignung des Mittenecker-Zeigeversuchs (MZV) für klinische Langzeitstudien und zur Validität seiner Testparameter [Experimental studies on the applicability of the Mittenecker Pointing Test in clinical long-term 
studies and the validity of its test scores]. Zeitschrift für Klinische Psychologie, 14, 130-144.

Shannon, C. E., \& WeaVer, W. (1949). The mathematical theory of communication. Urbana: University of Illinois Press.

Stoffers, D., Berendse, H. W., Deijen, J. B., \& Wolters, E. C. (2001). Motor perseveration is an early sign of Parkinson's disease. Neurology, 57, 2111-2113.

Stoffers, D., Bosboom, J. L. W., Deijen, J. B., Wolters, E. C., BeRENDSE, H. W., \& STAM, C. J. (2007). Slowing of oscillatory brain activity is a stable characteristic of Parkinson's disease without dementia. Brain, 130, 1847-1860.

TERAOKA, T. (1963). Some serial properties of "subjective randomness." Japanese Psychological Research, 5, 120-128.

Towse, J. N. (1998). On random generation and the central executive of working memory. British Journal of Psychology, 89, 77-101.

TOWSE, J. N., \& CHESHIRE, A. (2007). Random number generation and working memory. European Journal of Cognitive Psychology, 19, 374-394.
Towse, J. N., \& NeIL, D. (1998). Analyzing human random generation behavior: A review of methods used and a computer program for describing performance. Behavior Research Methods, Instruments, \& Computers, 30, 583-591.

TulviNG, E. (1962). Subjective organization in free-recall of "unrelated" words. Psychological Review, 69, 344-354.

WagenaAR, W. A. (1970). Subjective randomness and the capacity to generate information. Acta Psychologica, 33, 233-242.

\section{NOTE}

1. A copy of the MPT can be downloaded from the URL www.uni -graz.at/biologicalpsychology/mpt.html

(Manuscript received March 12, 2009;

revision accepted for publication September 6, 2009.) 